\title{
CORRECTION
}

\section{Correction to: Prognostic impact of sustained new-onset atrial fibrillation in critically ill patients}

Takuo Yoshida ${ }^{*}{ }^{\mathbb{D}}$, Shigehiko Uchino ${ }^{1}$, Yusuke Sasabuchi ${ }^{2}$, Yasuhiro Hagiwara ${ }^{3}$ and the AFTER-ICU study group

(C) 2020 Springer-Verlag GmbH Germany, part of Springer Nature

Correction to: Intensive Care Med (2020) 46:27-35 https://doi.org/10.1007/s00134-019-05822-8

The original version of this article unfortunately contained incorrect data for the SOFA score in the Table 1. The number of missing data of this score presented in the manuscript was also incorrect. Because the authors failed to add the score of renal part for calculating SOFA scores for each patient, the SOFA score in the primary manuscript was lower than the correct data unintentionally.
The SOFA score was not used in the multivariable analyses and was also not discussed in the manuscript. The authors apologize for the presentation of incorrect data. The corrected Table 1 can be found below. In the results section it should read: "Data were missing only for BMI (1 patient) and SOFA score (11 patients). 
Table 1 Demographic and clinical characteristics: non-sustained AF vs. sustained AF

\begin{tabular}{|c|c|c|c|c|}
\hline & $\begin{array}{l}\text { Overall } \\
(n=412)\end{array}$ & $\begin{array}{l}\text { Non-sustained AF } \\
(n=320)\end{array}$ & $\begin{array}{l}\text { Sustained AF } \\
(n=92)\end{array}$ & $p$ value \\
\hline Age, years & $75(67-81)$ & $74(66-80)$ & $76(67-83)$ & 0.106 \\
\hline Male sex, $n(\%)$ & $277(67)$ & $216(68)$ & $61(66)$ & 0.9 \\
\hline Body mass index, kg/m²a & $22(20-25)$ & $22(20-25)$ & $23(19-26)$ & 0.976 \\
\hline Hypertension, $n$ (\%) & $192(47)$ & $149(47)$ & $43(47)$ & 1 \\
\hline Diabetes, $n(\%)$ & $109(27)$ & $88(28)$ & $21(23)$ & 0.422 \\
\hline Congestive heart failure, $n(\%)$ & $43(10)$ & $36(11)$ & $7(7.6)$ & 0.438 \\
\hline Ischemic heart disease, $n(\%)$ & $43(10)$ & $37(12)$ & $6(6.5)$ & 0.181 \\
\hline Prior stroke or TIA, $n(\%)$ & $43(10)$ & $34(11)$ & $9(9.8)$ & 1 \\
\hline CHADS2 score & $1(1-2)$ & $1(1-2)$ & $1(0-2)$ & 0.838 \\
\hline Chronic hemodialysis, $n(\%)$ & $23(5.6)$ & $19(5.9)$ & $4(4.3)$ & 0.797 \\
\hline \multicolumn{5}{|l|}{ Previous medication } \\
\hline Calcium-channel blockers, $n(\%)$ & $135(33)$ & $106(33)$ & $29(32)$ & 0.802 \\
\hline$\beta$-Blocking agents, $n(\%)$ & $56(14)$ & $46(14)$ & $10(11)$ & 0.49 \\
\hline ACE inhibitors, $n(\%)$ & $22(5.3)$ & $19(5.9)$ & $3(3.3)$ & 0.433 \\
\hline ARBs, $n(\%)$ & $87(21)$ & $64(20)$ & $23(25)$ & 0.312 \\
\hline Antidiabetic agents, $n(\%)$ & $94(23)$ & $78(24)$ & $16(17)$ & 0.204 \\
\hline Anticoagulants, $n(\%)$ & $30(7.3)$ & $22(6.9)$ & $8(8.7)$ & 0.504 \\
\hline Antiarrhythmic drugs, n (\%) & $5(1.2)$ & $5(1.6)$ & $0(0)$ & 0.591 \\
\hline \multicolumn{5}{|l|}{ Patient category } \\
\hline Non-scheduled surgical, n (\%) & $94(23)$ & $71(22)$ & $23(25)$ & \\
\hline Scheduled surgical, n (\%) & $61(15)$ & $48(15)$ & $13(14)$ & 0.849 \\
\hline Medical, $n(\%)$ & $257(62)$ & $201(63)$ & $56(61)$ & \\
\hline APACHE II score at ICU admission & $23(18-29)$ & $23(17-29)$ & $24(19-28)$ & 0.553 \\
\hline SOFA at AF onset ${ }^{b}$ & $7(4-10)$ & $7(4-10)$ & $8(5-10)$ & 0.126 \\
\hline From ICU admission to AF onset, days & $1.6(0.7-3.0)$ & $1.6(0.7-3.1)$ & $1.7(0.7-2.8)$ & 0.972 \\
\hline Infection at AF onset, $n$ (\%) & $286(69)$ & $215(67)$ & $71(77)$ & 0.073 \\
\hline MV at AF onset, $n(\%)$ & $247(60)$ & $185(58)$ & $62(67)$ & 0.117 \\
\hline RRT at AF onset, $n(\%)$ & $101(25)$ & $79(25)$ & $22(24)$ & 1 \\
\hline
\end{tabular}

AF atrial fibrillation, TIA transient ischemic attack, CHADS2: one point: recent congestive heart failure, hypertension, age older than 75 years, diabetes mellitus; two points: transient ischemic attack or a prior stroke, $A C E$ angiotensin converting enzyme, ARBs angiotensin II receptor blockers, APACHE II Acute Physiology and Chronic Health Evaluation II, SOFA Sequential Organ Failure Assessment, MV mechanical ventilation, $R R T$ renal replacement therapy

a Missing data: one patient

b Missing data: eleven patients

\section{Author details}

${ }^{1}$ Intensive Care Unit, Department of Anesthesiology, Jikei University School of Medicine, Nishi-Shinbashi Minato-ku, Tokyo 105-8471, Japan. ${ }^{2}$ Data Science Center, Jichi Medical University, Tochigi, Japan. ${ }^{3}$ Department of Biostatistics, The University of Tokyo, Tokyo, Japan.

\section{Publisher's Note}

Springer Nature remains neutral with regard to jurisdictional claims in published maps and institutional affiliations.

Published online: 6 October 2020 